\title{
Philosophiques
}

\section{Pour une nouvelle lecture de la question de la " femme » : essai à partir de la pensée de Jacques Derrida}

\section{Linda Godard}

Volume 12, numéro 1, printemps 1985

URI : https://id.erudit.org/iderudit/203276ar

DOI : https://doi.org/10.7202/203276ar

Aller au sommaire du numéro

Éditeur(s)

Société de philosophie du Québec

ISSN

0316-2923 (imprimé)

1492-1391 (numérique)

Découvrir la revue

Citer cet article

Godard, L. (1985). Pour une nouvelle lecture de la question de la " femme » essai à partir de la pensée de Jacques Derrida. Philosophiques, 12(1), 147-164. https://doi.org/10.7202/203276ar
Résumé de l'article

Les propos de cet essai constituent une tentative de questionnement et de désamorcement de ce qui travaille souterrainement la mise en place des présupposés propres aux discours féministes. Cette entreprise aura au passage convoqué, interpellé - au moins - trois protagonistes principaux : Simone de Beauvoir, Luce Irigaray et Jacques Derrida. L'étonnement qui peut surgir face à cette rencontre inattendue ne saurait égaler celui qui survient devant la possibilité d'une nouvelle lecture de la question de la femme, autre que celle qui alimente, nourrit les discours des deux auteures précédemment mentionnées. Possibilité qu'ouvre le texte derridien et qui ici tente de se dire. 


\title{
POUR UNE NOUVELLE LECTURE DE LA QUESTION DE LA « FEMME » : ESSAI À PARTIR DE LA PENSÉE DE JACQUES DERRIDA
}

\author{
par Linda Godard
}

\begin{abstract}
RÉSUMÉ. Les propos de cet essai constituent une tentative de questionnement et de désamorcement de ce qui travaille souterrainement la mise en place des présupposés propres aux discours féministes. Cette entreprise aura au passage convoqué, interpellé - au moins - trois protagonistes principaux : Simone de Beauvoir, Luce Irigaray et Jacques Derrida. L'étonnement qui peut surgir face à cette rencontre inattendue ne saurait égaler celui qui survient devant la possibilité d'une nouvelle lecture de la question de la femme, autre que celle qui alimente, nourrit les discours des deux auteures précédemment mentionnées. Possibilité qu'ouvre le texte derridien et qui ici tente de se dire.
\end{abstract}

ABSTRACT. This essay aims at revealing and examining elements which, beneath the surface, create the organization of presuppositions which are characreristic of feminist discourses. In the process, this work will have called forth - at least - three principal protagonists : Simone de Beauvoir, Luce Irigaray and Jacques Derrida. The surprise which this unexpected grouping might provoke can not equal the astonishment which springs from the possibility of a new reading of the "féminine " question, - a reading which differs from that which nourishes the discourses of the first two authors mentionned. It is Derrida's text which creates this possibility and which attemps to express itself here.

À la lecture du texte de Jacques Derrida Éperons : les styles de Nietzsche, on voit s'opérer un bouleversement quant à la manière de penser le "féminin " et cela ne va pas sans avoir quelque impact sur la façon d'envisager la littérature féministe contemporaine. Les quelques propositions rencontrées dans le parcours de ce texte - qui ne se donnent jamais simplement comme telles — sont tout à fait étrangères à celles qui prédominent 
actuellement dans les discours féministes, qu'il s'agisse des discours qui insistent sur l'aspect socio-historique des revendications féministes et qui, dans le même temps, entretiennent un lien très intime avec les thèses de Marx quant à la libération des prolétaires, ou bien qu'il s'agisse des discours qui mettent plutôt de l'avant la question de la différence sexuelle et qui insistent par conséquent, sur le fait que ce qui constitue la spécificité des femmes est essentiellement irréductible à ce qui constitue la spécificité des hommes. Discours qui non seulement affirment l'irréductibilité d'une spécificité féminine mais qui, en outre, sur-valorisent cette dernière au détriment de celle qui est dite «propre » aux hommes. Le discours de Jacques Derrida engage, quant à lui, la mise en question des conceptions du " féminin " au sein de ces discours et il nous invite à penser autrement, par des effets de déplacement, le « féminin ». Cette pensée autre n'est pas sans aller de pair avec la question du style, en tant que question de l'écriture : «La question du style, comme question de l'écriture, devient plus puissante que tout contenu, tout sens, plus puissante que toute différence sexuelle ${ }^{1}$.

Dès lors, je me propose dans cet essai de mettre en relief ce qui s'annonce dans le texte derridien comme une façon autre de penser le "féminin ». Afin de voir, par ailleurs, ce que cela engage pour la question de l'écriture ou, c'est la même chose, la question du style, vue dans son rapport au "féminin ». Cette mise en relief s'effectuera en indiquant le tracé respectif de chacun des types de discours féministes précédents, ce qui permettra de voir comment ils ont identifié le "féminin » et la «femme » ou la " féminité » ou encore la « sexualité féminine ». Et comment aussi, ce faisant, chacun a entouré, enveloppé de certaines déterminations la question de la " sexualisation " de l'écriture ou, dit autrement, la question de l'établissement d'une distinction puis de l'attribution d'une différence de nature entre les écrits (et discours) «masculins» et les écrits (et discours) "féminins". Je fréquenterai, dans la mise en place des propositions de ces discours, deux principaux ensembles de textes :

1. Jocelyne Ouimet, Le procès du propre, Thèse de doctorat, Avril 1982, UdM, p. 194. 
tout d'abord, le texte de Simone de Beauvoir - Le deuxième sexe ${ }^{2}$ - qui apparaît comme un excellent représentant du type de discours féministes où se précipitent des questions telles que le rôle et la place de la femme dans les structures économico-politicosociales d'un système de production donné. Puis ceux de Luce Irigaray - particulièrement la seconde partie de son Speculum de l'autre femme ${ }^{3}$ - où il est question d'une certaine mise en lumière du processus de métaphorisation à l'œuvre dans le "Mythe de la caverne » tel qu'il a germé dans l'imaginaire platonicien. Mise en lumière qui se fait simultanément par une certaine mise en place d'un autre processus de métaphorisation, soit celui propre à l'imaginaire irigarayien. Je tenterai de voir, au cours de cet essai, en quoi consiste ce processus, à quoi il renvoie et ce que cela a pour effets dans la façon de concevoir le «féminin", la question de l'écriture et celle du style. Ce texte sera mis en rapport avec «Le prix de la sérénité » où, comme on le verra, l'on retrouve le même type de procès métaphorique que le texte précédent. ${ }^{4}$

\section{LE DISCOURS FÉMINISTE DE SIMONE DE BEAUVOIR ; SON ARGUMENT FONDAMENTAL.}

Commençons donc par le texte de Simone de Beauvoir, où l'on peut retenir comme thèse principale cette proposition : la libération des femmes doit passer d'abord et avant tout par leur égalité économique, sociale et politique avec les hommes : "C'est par le travail que la femme a en grande partie franchi la distance qui la séparait du mâle; c'est le travail qui peut seul lui garantir une liberté concrète " ${ }^{5}$. C'est à l'intérieur même du système de production capitaliste que les sujets féminins doivent lutter pour la réalisation de leur liberté, indissociablement liée à l'indépendance économique qui pour eux en découle. Évidemment, celle-ci ne pourra se réaliser véritablement que dans la transformation de ce système de production, caractérisé essentiellement par la domination et l'exploitation du Capital sur le pro-

2. Simone de Beauvoir, Le deuxième sexe, Tome II, Éd. Gallimard, 1949, Paris.

3. Luce Irigaray, Spéculum de l'autre femme, Éd. de Minuit, Paris, 1974, 463 pages.

4. Luce Irigaray, extrait de "L'Oubli de l'air, lecture de Heidegger, " in Martin Heidegger, Éd. de l'Herne, Oct. 1983, Paris.

5. Simone de Beauvoir, op. cit., p. 521. L'italique est de nous. 
létariat, en un autre système de production, où ces rapports de domination et d'exploitation seraient abolis. La réalisation des visées de ce féminisme est intimement liée au problème que pose à Marx la question du passage d'un monde où prédomine l'organisation matérielle et les représentations idéologiques du système de production capitaliste à un monde où prévau(drait)t l'organisation matérielle et les représentations idéologiques du système de production socialiste, "réalité " à créer, caractérisée essentiellement par la fin des inégalités sociales. Et il se trouve que, dans un certain mouvement d'identification au marxisme, le discours de Simone de Beauvoir n'y puise pas qu'une orientation politique donnée, il s'y voit, par ce geste, subordonné et dominé, puisque Marx, ne considérant pas comme telle la question de la différence sexuelle, se trouve à la subordonner à celle de la lutte des classes. Ainsi, ce discours tend non seulement à réduire la multiplicité et la diversité du féminin à un sujet collectif mais de plus il tend à ramener ce sujet collectif féminin au sujet universel de Marx, soit le prolétaire. Et bien que, dans une entrevue récente accordée par Mme de Beauvoir à deux journalistes de la revue La vie en rose $e^{6}$, l'on puisse lire qu'elle aurait changé d'avis au sujet de la lutte des femmes : « (. . .) notamment (qu')elle a cessé de croire qu'il fallait subordonner celle-ci à la lutte des classes, et que le socialisme mettrait fin comme par magie au sexisme $»^{7}$, un rapide coup d'œil sur la littérature féministe du $\mathrm{XX}^{\mathrm{e}}$, siècle indique que la conception beauvoirienne de la femme, du "féminin ", caractérisée essentiellement par une tension entre les deux réductions signalées plus haut, a eu une influence considérable. Elle a donné naissance, en effet, à de nombreux écrits où il est question de travailler à la constitution d'une prétendue « histoire » des femmes qui serait demeurée méconnue et occultée par celle de l'humanité ou, plus précisément, celle des hommes. Il me paraît donc important d'interroger cette conception, que l'auteure d'origine continue d'y adhérer ou non.

\footnotetext{
6. "Simone de Beauvoir, féministe " dans la La vie en rose, Mars 1984, no. 16, Montréal, pp. $25-44$.

7. Ibid., p. 41 .
} 
II. ÉTROITE ASSOCIATION ENTRE LA QUESTION DU « FÉMININ », LA CONDITION SOCIALE DES FEMMES E'T

UNE ÉCRITURE DITE « FÉMININE ».

Depuis la parution de cet essai (1949) et jusqu'à aujourd'hui, il est symptomatique de relever les thèmes qui ont traversé les écrits des femmes se réclamant ou s'inscrivant dans le sillage du féminisme disons "socio-historique ": la pornographie, le salaire aux travaux ménagers, la contraception, l'avortement, la violence et le viol, les "progrès " scientifiques et techniques, l'amour, les modèles féministes et l'ambition des femmes, la création littéraire. Ces différents thèmes peuvent se ramener, si on y regarde de plus près, à deux idées principales : le rapport des femmes au travail et celui à leur sexualité. Les écrits qui s'articulent autour de ces thèmes ou "idées " se sont voulus (se veulent) des écrits "féminins ", des écrits qui affirment quelque chose comme une vérité de la femme, une identité féminine à laquelle, cela va de soi, toutes les femmes devraient se sentir conviées. Et l'affirmation de cette "vérité " ou de cette "identité " de la femme n'est pas sans entretenir quelque rapport avec des questions telles que le rôle et la place de la femme dans l'histoire de l'" humanité ". Pour Simone de Beauvoir, ainsi que pour les femmes qui se disent ses "filles spirituelles ", la question du "féminin » est étroitement associée à la condition sociale de la femme. Et, par voie de conséquence, leurs discours inciteront les femmes à s'engager dans un processus d'identification où les différences importantes qui existent entre elles seront subordonnées, secondarisées par rapport au but, à l'objectif commun qui les unissent, soit l'abolition de la société capitaliste et patriarcale qui les a confinées à n'être que l'ombre, le négatif de l'homme. Et comment cela pourra-t-il se faire ? D'abord, à travers l'affirmation de leur égalité avec les hommes, égalité rendue possible, selon Simone de Beauvoir, que si les femmes réalisent les conditions sociales leur permettant d'être indépendantes économiquement des hommes, ce qui entraînera (devrait entraîner) leur autonomie de pensée. Et cette indépendance économique et idéologique (on reconnaît ici le schème de pensée propre à Marx où l'économique, la base matérielle de la " réalité » historique, déterminerait, en dernière instance, la sphère de l'« idéologique », ou, dans un langage emprunté aux contemporains/nes, du « symbolique ». Cela, on le verra plus loin, aura 
des conséquences dans la façon dont la pensée beauvoirienne conçoit la question du "féminin " dans son rapport à celles de l'écriture et du style) ne peut s'accomplir que par l'intermédiaire de la réalisation de deux conditions : que les femmes puissent se libérer des contingences liées à leur processus de reproduction naturelle et qu'elles puissent trouver, avoir leur place au sein du processus de production sociale, par la voie du travail. Car pour Mme de Beauvoir, l'aliénation de la femme consiste dans le fait de sa subordination à l'espèce : "C'est par la maternité que la femme accomplit intégralement son destin physiologique (. . . ) puisque tout son organisme est orienté vers la perpétuation de l'espèce ; la femme, comme l'homme, est son corps, mais son corps est autre chose qu'elle ${ }^{8}$. En se libérant des contingences liées à ce qui la détermine physiologiquement, la femme pourrait ainsi choisir ses rapports avec autrui. Cette prise de position soustend tout un système de valeurs propre à de Beauvoir et selon lequel « (. . .) l'individu doit l'emporter sur l'espèce, l'esprit sur le corps, le choix sur la contingence, la nécessité sur la gratuité » ou dit dans les termes de la " morale existentialiste ", "la transcendance sur l'immanence " ${ }^{9}$. La maîtrise de cette part de déterminations, qui confine les femmes aux tâches relevant de leur «destin " physiologique, leur permettra de disposer d'un temps où elles pourront travailler, s'instruire, voyager. Ces thèmes du temps et de l'anti-maternel sont très importants dans le discours beauvoirien car ce sont eux qui déterminent le type de rapports que les femmes seraient susceptibles d'entretenir avec leur(s) vie(s). Car identifiant la femme aux déterminations physiologiques de son corps, donc à la mère, et l'homme à l'esprit équation qui n'est pas sans présenter quelque air familier Simone de Beauvoir se (nous) précipite dans une conception de la femme où l'étalon de mesure est l'homme occidental, ses pouvoirs, ses actions, sa liberté, sa société. Ainsi, pour elle, la libération de l'aliénation, qu'entraîne la spécificité biologique de la femme, passera par une importance démesurée accordée à l'agir, à l'activation, à l'activisme de l'être « humain » dans/ sur le monde, où il ne faut jamais s'arrêter, où il faut « (. . .)

8. Simone de Beauvoir, op. cit., Tome II, p. 135 ; Tome I, p. 46, cité par Nancy Huston dans "Simone de Beauvoir, féministe" (voir note 6 ci-dessus).

9. Simone de Beauvoir, op. cit., tome I, p. 42. 
emporter du travail partout avec soi ; faire coïncider le plus étroitement possible la vie et le travail (. . .) ${ }^{10} \ldots$. comme les hommes, comme Sartre qui lorsqu'il nous parle d'elle, ou elle de lui, c'est dans des termes qui réduisent à zéro l'altérité. Et cela n'est pas sans entretenir quelque lien, il me semble, avec la façon dont de Beauvoir envisage la libération des femmes sur le plan social. En effet, pour elle, "C'est au sein d'un monde donné qu'il appartient à l'homme de faire triompher le règne de la liberté ; pour remporter cette suprême victoire il est entre autres nécessaire que par-delà leurs différenciations naturelles hommes et femmes affirment sans équivoque leur fraternité ${ }^{11}$.

Donc, sur le plan d'un système de valeurs personnel, la logique beauvoirienne prend appui sur les préceptes sartriens, à la limite elle s'y identifie, et cela n'est pas sans influencer sa façon de concevoir la libération des femmes sur le plan social : ainsi, d'une part, il y a réduction de l'altérité au même "Sartre était un autre moi-même " dira Simone de Beauvoir et réciproquement, elle est (fut) pour lui l'armature de sa vie, sa conscience et sa raison, "Tout ce que je suis de bien, c'est à cause de vous que je le suis " (Sartre in "Lettres au Castor ") - et, d'autre part, il y a affirmation de la nécessité du dépassement de la différence sexuelle dans la lutte qui doit être menée pour faire triompher le règne de la liberté dans le monde.

III. CONSÉQUENCES DES PRISES DE POSITION BEAUVOIRIENNES SUR LA CONCEPTION DE L' « ÉCRITURE " ET DU «STYLE ".

Quelles conséquences ces prises de position peuvent-elles avoir sur la façon d'entrevoir les questions de l'écriture et du style dans leur rapport à celle du " féminin " ? À quelles déterminations particulières cela nous conduira-t-il dans la considération de ces questions ? Disons tout d'abord que la " féminité " ou la « masculinité » de l'écrit serait le fait de la différence qui existe entre la condition de la femme et celle de l'homme. Ainsi, pour Simone de Beauvoir, il n'y a pas de différence comme telle entre l'écriture masculine et l'écriture féminine lorsque cette question est considérée dans son strict rapport au langage. La

10. Ibid., p. 43.

11. Simone de Beauvoir, op. cit., tome II, p. 577. L'italique est de nous. 
question de la différence sexuelle est déportée ailleurs, à un autre niveau, celui de la condition de la femme et de l'homme, qu'expriment le contenu et le style de l'écrit :

(. . .) je suis tout à fait contre les femmes qui cherchent une écriture "féminine ». Le langage est un outil comme les autres, il a été forgé par ce monde et il se trouve que le monde a été masculin. Mais maintenant, il faut plutôt voler l'outil que le transformer. Ce qu'il y a de différent, c'est la condition de la femme qui n'est pas la même que l'homme. Un livre exprime d'abord une condition : alors, en effet, un écrit féminin n'est pas le même qu'un écrit masculin, quant au contenu et quant au style. Mais je ne pense pas qu'il y ait vraiment une écriture, un langage qui doivent être différents ${ }^{12}$.

Ainsi, quasi naïvement, la question de la différence sexuelle, celles de la "féminité ", de la "sexualité féminine ", de la « femme » émigrent du côté de la condition humaine qui porte toujours, déjà la marque de déterminations socio-historiques. Ainsi la question du style n'est pas dissociée de celle du contenu, du sens et elle est tributaire de la différence sexuelle qui se joue au niveau des conditions sociales respectives de la femme et de l'homme. Et en ce qui concerne la femme, cette condition sociale particulière, qui se caractérise, pour l'essentiel, par un état d'infériorisation par rapport aux hommes, sera directement reliée, dans le discours beauvoirien, au fait que les femmes « (...) sont femmes par leur structure physiologique ; aussi loin que l'histoire remonte, elles ont toujours été subordonnées à l'homme : leur dépendance n'est pas la conséquence d'un événement ou d'un devenir, elle n'est pas arrivée. (. . .) La division des sexes est en effet un donné biologique, non un moment de l'histoire humaine ${ }^{13}$. Et en dépassant ce "donné biologique ", la femme pourra commencer à prendre part à l'élaboration du monde, à celle de son " histoire ". Ici se glisse un paradoxe, celui sur lequel insiste, notamment, Jacques Derrida dans une entrevue parue dans la revue "Diacritics " ${ }^{14}$ et dont je vais tenter de relater ici l'essentiel, afin de le mettre en rapport avec le discours de de Beauvoir.

12. La vie en rose, p. 32-33.

13. Ibid., p. 34.

14. Jacques Derrida et Christie V. McDonald, "Choreographies " in Diacritics, Vol. 12, The Johns Hopkins University Press, 1982, p. 66-76. 


\section{LE « FÉMININ » CHEZ JACQUES DERRIDA ET LA TENDANCE FÉMINISTE SOCIO-HISTORIQUE.}

Voulant faire émerger une (l') histoire de la femme qui aurait été passée sous silence par celle de l'homme, une certaine orientation idéologique au sein du mouvement féministe a voulu la donner comme constituant la "véritable ", la « réelle " histoire de toutes les femmes, affirmant par le fait même une vérité ou une identité de la différence sexuelle et de la féminité. Or, pour Jacques Derrida, "Il n'y a pas de vérité de la femme (. . .) (et) (. . .) c'est parce que cet écart abyssal de la vérité, cette non-vérité est la "vérité ". Femme est un nom de cette nonvérité de la vérité " ${ }^{15}$. "(. . .) si la femme " est " vérité, " elle» sait qu'il n'y a pas la vérité, que la vérité n'a pas lieu et qu'on n'a pas la vérité. Elle est femme en tant qu'elle ne croit pas, elle, à la vérité, donc à ce qu'elle est, à ce qu'on croit qu'elle est, que donc elle n'est pas ${ }^{16}$. Ainsi pour lui, l'élaboration, la constitution que font certaines féministes d'une histoire occultée, méconnue et qui serait proprement "féminine » n'est qu'un renversement qui revient au même, puisque le concept d' " histoire " véhicule, porte en lui les notions de " progrès ", d'étapes déterminées et est ultimement commandé par une " fin ", un « télos ». À ses yeux, les femmes devraient renoncer à toute vue progressiste dans leurs évaluations de l'histoire, car cela a un impact immédiat sur la façon de concevoir le " féminin ». N'établissant pas une distinction entre « (. . .) ce qui est "féminin " et la "féminité ", la fémini « té " de la femme, la sexuali « té " féminine et autres fétiches essentialisants " ${ }^{17}$, elles peuvent produire l'équation entre écrits " féminins » et écrits dénonçant la condition sociale des femmes, entachée, quant à elle, d'un certain déterminisme biologique comme on a pu le voir. Et dans ces écrits, il est question de rechercher la place de la femme dans ce monde qui jusqu'à maintenant est dit avoir été construit par et pour les hommes. Mais pourquoi ne pas porter quelque attention à l'interrogation derridienne quant à ce problème de la place de la femme : "Why must there

15. Jacques Derrida, Éperons : les styles de Nietzsche, Flammarion, 1978, p. 39. L'italique est de nous.

16. Ibid., p. 40.

17. Ibid., p. 43 . 
be a place for woman ? And why only one, a single, completely essential place ? (. . .). (. . . ) there is no one place for woman"18. Cette interrogation/prise de position derridienne ne concerne pas que les présupposés idéologiques du discours beauvoirien et ceux de ses " filles spirituelles» (telles que certaines féministes se nomment, se désignent), mais a aussi rapport avec ceux du discours irigarayien. Mais avant d'aborder le second pan de cet essai, je ferai un bref retour sur la conception de l'écriture de Simone de Beauvoir en la mettant en relief avec celle (si encore tel terme, telle expression peut avoir quelque pertinence dans l'approche du texte derridien) de Derrida où comme il a été signalé au début de ce texte, la question de l'écriture, en tant que question du style, « devient plus puissante que tout contenu, tout sens, plus puissante que toute différence sexuelle ».

\section{LE TRAVAIL DE DÉPLACEMENT CONCEPTUEL CHEZ DERRIDA DANS SON RAPPORT À LA CONCEPTION DU « FÉMININ " CHEZ DE BEAUVOIR.}

Identifiant ses positions théoriques à celles des discours où l'Un se subordonne le multiple, la nécessité la contingence, l'esprit le corps ou, c'est la même chose, la matière l'esprit, elle enregistre une conception de la question de l'écriture et de celle du style où une soi-disant " réalité ", ici la condition sociale des femmes, déterminerait en dernière instance (?!) ce qui travaille symboliquement l'écriture des femmes, assimilée, ramenée ici à l'écriture "féminine ". On est ainsi renvoyé, confiné à l'opposition du féminin et du masculin, qui a pour point d'ancrage les différences qui existent entre les conditions sociales respectives de la femme et de l'homme et qui ne sont pas sans entretenir quelque intime lien, dans un premier temps, à la différence sexuelle en tant que division des sexes considérée comme étant un donné biologique. Derrida, pour sa part, en dé-centrant la question du « féminin » par rapport aux déterminations essentialistes qui entourent ce concept dans la logique dichotomique, oppositionnelle de la métaphysique occidentale, nous convie à réfléchir ce problème comme étant ce qui permet la "mouvance" de l'écriture en tant que question du style. Cette

18. Jacques Derrida et Christie V. McDonald, op. cit., p. 68. 
question en tant que telle, ne peut donc plus se rapporter à un propre, à une essence, à un contenu, à un sens. Elle les déborde de toute part. Elle devient plus puissante que toute différence sexuelle, que toute division des sexes, contrairement à la conception de de Beauvoir qui précipite la question du style du côté de la contingence alors que celle de l'écriture (par le biais du langage) est gardée à l'abri du côté de la nécessité, de toute contamination corporelle, anatomique, sexuelle. Chez Derrida, il n'y a pas ce régime propre à la pensée dichotomique du « logo-phallo-centrisme » occidental et le « féminin » n'est donc pas approché - « il » ne peut pas être simplement approché, l'on verra, un peu plus loin, pourquoi ou plutôt de quelle manière - dans un rapport oppositionnel au " masculin ». Mais plutôt par des effets de déplacement, de décentrement qui, tout d'abord, opèrent la distinction entre la question du «féminin » et tout ce qui détermine, connote cette notion, soit les «fétiches essentialisants " que sont la féminité, la fémini « té », la sexuali « té » féminine. Tentant de la dégager de tout déterminisme, à travers une pensée de la différé(a)nce, Derrida la mettra en rapport avec une nouvelle conceptualité permettant de penser autrement la question de l'écriture en tant que question du style, question indissociablement liée au rapport de la femme à l'art et à la vérité. « Mais la simple formation de cette problématique commune suspend la question « qu'estce que la femme ? ". On ne peut plus chercher la femme ou la féminité de la femme ou la sexualité féminine. Du moins ne peut-on pas les trouver selon un mode connu du concept ou du savoir, même si on ne peut s'empêcher de les chercher» ${ }^{19}$, ajoutera-t-il très expressément. Ainsi il n'opère pas qu'un simple renversement de la question de la "femme" mais de plus il crée, invente de nouveaux concepts qui permettront de penser autrement, de jeter un nouvel éclairage sur cette question, notamment le concept de "différance ", à la suite duquel se présentent ceux de « dissémination", de " trace ", de « supplément ", d' « bymen", de "pharmakon", de "double invagination". Ces concepts (sans que le mot « concept » soit entouré de toutes les déterminations habituelles. Remarquons qu'ici surgit un des problèmes qui caractérise la démarche derridienne, à savoir le fait que par des effets de déplacement, ou de dé-centrement - comme, par

19. Jacques Derrida, op. cit., p. 57. 
exemple, dégager la question du féminin de tout ce que ce terme recouvre, renferme de délimitations, de caractérisations propres, ou dites telles, à l'être biologique et socialisé appelé "femme " - elle (il) introduit une façon autre de penser une question, mais dans le même temps, cette dernière porte toujours, déjà la marque, l'empreinte, la trace de ce de quoi elle tente de se dégager, de s'éloigner. Difficile sortie de la logique dichotomique surtout lorsqu'on reprend des termes comme le « féminin " et le «masculin » qui recèlent en leur sein une surcharge de connotations. Mais ici se glisse la question du style. On y reviendra.) seront proposés pour penser autrement que la logique binaire où l'altérité est toujours subordonnée et réduite au même. Logique de la « différance » qui renvoie à un temps autre l'accomplissement, la réalisation, l'achèvement du processus de différenciation qui produirait l'opposition dans le deux. Les repoussant sans cesse de telle sorte qu'ils n'adviennent jamais. Logique où le masculin peut être pensé comme étant constitutif du féminin, et inversement. Où l'un (l'autre) travaille incessamment l'autre (l'un), sans jamais que l'un (l'autre) puisse être séparé, dissocié de l'autre (l'un) : " (. . .) on ne peut séparer l'bomme de la femme, les opposer comme deux figures distinctes ayant leur identité propre. De l'bomme et de la femme, il n'y a plus d'opposition, il y a la "différance ". La structure de la "différance " ne peut se dissocier d'une certaine altérité plus irréductible encore que celle que l'on prête à l'opposition ${ }^{20}$ et s'approcher de la question du «féminin » sera impossible « (. . .) sans se soustraire à la logique de l'identité, voire de la dialectique de la contradiction. La syntaxe à laquelle nous soumettons le discours sur la femme se soustrait aussi à toute différence sexuelle. La femme peut être aussi bien l'écriture, une parole, une idée. L'écriture s'associe à tout ce qui est féminin ${ }^{21}$. C'est-à-dire qu'elle s'associe à tout ce qui relève de l'« opération féminine " dont il faut se tenir à distance et qui dans l'écriture, notamment celle de Nietzsche, va contraindre "à la mise entre guillemets de la "vérité " - et par suite rigoureuse de tout le reste - ", qui, donc, "va inscrire la vérité - et par suite rigoureuse, inscrive en général ». C'est à elle que revient le style qui, par des écarts de plume, imprimera la loi de l'espacement, permettant

20. Jocelyne Ouimet, op. cit., p. 181.

21. Ibid., p. 197. 
ainsi de suspendre le rapport à un contenu, un sens, une (la) vérité. Et s'il faut se tenir à distance de cette " opération féminine ", de cette pratique stylet et stylée qui elle-même produit de la distance « (. . .) c'est que " la femme » n'est peut-être pas quelque chose, l'identité déterminable d'une figure qui, elle, s'annonce à distance, à distance d'autre chose et dont il y aurait à s'éloigner ou à s'approcher ${ }^{22}$. Dès lors, on peut se demander comment a-t-il pu se produire que, ayant enregistré, pris en compte, voire approprié les différentes propositions que formule Jacques Derrida sur la question du "féminin » et celle de l'« écriture » ou « style » qui s'y rattache où il est, entre autres et conséquemment avec la proposition précédente, clairement dit que «De même qu'il n'y a pas d'être ou d'essence de « la » femme ou de «la » différence sexuelle, il n'y a pas d'essence du « es gibt " dans le « es gibt Sein ", du don et de la donation de l'être ${ }^{23}$, Luce Irigaray ait ancré la question de l'« écriture » ou "style " dans un processus de métaphorisation où l'anatomie spécifique de la femme, sa jouissance particulière, les fonctions singulières de son corps constituent la matière première de symbolisation? Je tenterai, dans l'approche de cette question, d'indiquer quelques signes qui nous permettraient d'entrevoir l'adéquation qu'elle fait entre le motif de la « différance » et celui d'une spécificité " féminine " - ce dernier étant inscrit dans la question de la « différence sexuelle » - pour ensuite en voir les effets sur la façon de concevoir le " féminin » dans son rapport à la question de l'« écriture » ou du « style ».

VI. L'APPROCHE DE LUCE IRIGARAY DE LA QUESTION DU «FÉMININ », DE L' « ÉCRITURE » ET DU «STYLE » OU LE JEU D'ÉTRANGES ANALOGIES.

Luce Irigaray tente de débusquer, d'ébranler dans une manière de vertige toute derridienne, les discours philosophiques mâles où elle repère, pour les déconstruire, les représentations (ou figures) féminines. Mais, ce faisant, elle met aussi en place un autre processus de métaphorisation où l'opposition du sexe de la femme au dieu phallus conditionne, détermine son rapport au « symbolique ». Par exemple, la mise en lumière

22. Jacques Derrida, op. cit., p. 38.

23. Id., p. 100 . 
du procès métaphorique à l'œuvre dans le « Mythe de la caverne » platonicien-où, dit trop rapidement, on aurait quelque chose comme un schéma qui renverrait à la symbolisation de la pénétration de la lumière du soleil (qui représente ici la connaissance, le savoir des hommes-philosophes) dans l'antre caverneux où sont enchaînés les esclaves - se joue dans une interprétation où l'auteure associe ce schéma à ce qui, dans les discours philosophiques des hommes, est (a été) réprimé, refoulé, voire oublié : le corps, notamment celui de la femme, de la femme-mère. Cet oubli, celui d'un chemin - celui menant du dehors au dedans, de la lumière à l'obscurité, de la réalité au rêve, bref chemin menant ou plutôt signalant le leurre, l'illusion d'une pensée dichotomique, oppositionnelle ou, c'est la même chose, d'une logique du même - est aussi l'oubli, analogiquement, d'un autre passage (ou du même passage ?), le vagin de la femme, "Vagin oublié - passage manquant, laissé pour compte, entre le dehors et le dedans, entre le plus et le moins, . . . . ${ }^{24}$. Indice ici donc du type de procès de métaphorisation de Luce Irigaray qui consiste à établir d'abord des liens entre la façon dont les discours philosophiques des hommes se constituent (se sont constitués) et la question de la "femme " - ici identifiée au corps de la femme - qui, souterrainement, la travaille, en est condition de possibilité, pour ensuite renverser cet ordre discursif. Renversement qui s'opère par l'exploitation et la revalorisation d'une certaine spécificité féminine et qui rend possible « un recouvrement du féminin dans le langage ». Luce Irigaray participe d'un courant du mouvement des femmes en France où le mot «Différence " recouvre cette tentative de recherche d'une spécificité féminine, presque toujours commandée par certaines déterminations propres au "corps » de la femme : règles, grossesses, accouchements, temps cyclique et son éventuel retentissement dans le «corpus» littéraire. Ainsi la question du «féminin » ou le motif de la « différance » sont associés, voire assimilés à la femme, à la fémini « té », à la sexuali « té » féminine. Poursuivons plus avant afin de voir de quelle façon cela se joue dans le discours irigarayien, en mettant notamment en rapport le texte qui vient d'être brièvement abordé, où l'on peut retenir cette relation entre l'oubli d'un passage « entre » et celui

24. Luce Irigaray, Speculum (. . .), op. cit., p. 306. 
du sexe de la femme, plus précisément de sa sexualité, et un autre texte - plus récent - d'Irigaray auquel j'attacherai maintenant quelque importance, «Le prix de la sérénité ». Cette dernière nous rappelle ici l'oubli qu'aurait fait Heidegger d'un certain " passage vers », alors que lui-même est si absorbé par le problème que pose à l'humanité l'oubli de la question de « l'Être ». Préoccupé dans la construction d'un pont qui garantit la présence de "l'Etre-soi » à soi et au monde, en l'éloignant du néant, de la mort, de l'absence, du rien, de l'« Autre (de soi) », Heidegger a oublié (a produit l'oubli du) le passage qui conduit vers l'autre, «le il y a du pont a emporté dans sa construction l'autre vers qui il se voulait passage " ${ }^{25}$. La brèche qu'ouvre Luce Irigaray dans le texte heideggerrien vise à repérer et à dénoncer cet oubli en indiquant à quoi d' « autre », plutôt qu'au rien, à l'absence, au néant, (. . .) il renvoie (renverrait). L'équation est étrangement simple : cet oubli, dans le processus de métaphorisation " propre " à Heidegger, serait celui de l'air, plus précisément celui que la mère - d'abord - donne et ceci sans retour. Et «Si cet air est - d'abord - la matière fluide véhiculée par le sang qu'elle donne, il peut s'entendre aussi de la voie et du phénomène. Ils en proviennent, et sont la possibilité - encore matérielle - de l'appellation-dénomination, de l'apparaître en présence ${ }^{26}$. Ainsi le discours d'Irigaray, le processus de métaphorisation qui en est constitutif, n'est pas sans porter la trace, l'empreinte de quelque positivité - la question du « féminin » étant indissociable (contrairement à Derrida) de la question d'une spécificité "féminine »-, de quelque structure formelle où les sujets-femmes reçoivent la constellation de la prédication "féminine »: la féminité, la fémini « té », la sexuali « té " féminine. D'un habile tour de main, Irigaray semblait avoir ébranlé, une fois pour toutes, l'ingénieuse et complexe pensée heideggerrienne, sauf que, dans le même temps où elle déconstruit celle-ci, elle construit, si l'on peut dire, la sienne. Tout en articulant un discours, qui se veut discours de l'Altérité, de la «Différence » (de la « différance ») - où l'on peut relever, notamment, que ce motif habite, meut le «style » ou " écriture " d'Irigaray, et que cela, pour elle, ne contredit pas

25. "Le mix de la sérénité ", op. cit., p. 155.

26. Id., p. 158. Je souligne. 
le fait qu'elle ancre, par ailleurs; son " dire " littéraire dans un processus de métaphorisation où pré-dominent, où sont survalorisées les représentations, les images que la (les) femme(s) crée(nt), invente(nt), construise(nt) à partir de la relation qu'elle(s) entretien(nen)t à son (leur) corps. Du corps on passe au « corps propre ", à la question du « corpus littéraire » qui se joue dans un rapport à la « différence sexuelle»-Luce Irigaray, comme d'un tremplin, part de ce tissu symbolique, qui prend appui sur de très précises déterminations physiologiques, propres à la femme (il faudrait voir, à cet effet, comment fonctionnent les déplacements qu'elle tente de faire d'une conceptualité « logophallo-centrique ", cette part derridienne en elle présente dans son "style " ou " écriture ", compte tenu qu'elle entoure précisément ces questions de tout un ensemble de déterminations qui se réferent à une spécificité féminine) pour établir un rapport entre l'image que suscite en elle le jeu des lèvres du sexe de la femme, qui continuellement, incessamment se retouchent, et la question du "style » ou " écriture ". Consciente du fait que les motifs du «se toucher " et de la «proximité " pourraient, isolés comme tels ou réduits en énoncés, " effectivement passer pour une tentative d'approprier le féminin au discours ", singulièrement dans le traitement qu'elle fait de la question du " style » ou « écriture » de la femme - car ceux-ci ont participé à l'édification, la construction de l'image, de la métaphore du jeu des lèvres du sexe de la femme - elle n'en reprend pas moins les termes pour caractériser ces deux questions car même si cela était il « Resterait à éprouver si «ce toucher»- - ce toucher - le désir du proche plutôt que du propre, etc., n'implique pas un mode d'échange irréductible à tout " centrage ", " centrisme ", étant donné la façon dont le "se toucher " de l'« auto-affection " féminine joue comme un renvoi de l'un(e) à l'autre sans arrêt possible, et que la proximité y confond toute adéquation, appropriation $"{ }^{27}$, car c'est en la femme même qu'a lieu le mouvement de la "différance ". "Ainsi, par exemple, l'auto-érotisme de la femme est-il très différent de celui de l'homme. Celui-ci a besoin d'un instrument pour se toucher: sa main, le sexe de la femme, le langage . . . Et cette autoaffection exige un minimum d'activité. La femme, elle, se touche

27. L. Irigaray, Ce sexe qui n'en est pas un, Éd. de Minuit, Paris, 1977, p. 76. 
d'elle-même et en elle-même sans la nécessité d'une médiation, et avant tout départage possible entre activité et passivité. La femme "se touche" tout le temps, sans que l'on puisse d'ailleurs le lui interdire, car son sexe est fait de deux lèvres qui s'embrassent continûment. Ainsi, en elle, elle est déjà deux - mais non divisibles en un(e)s - qui s'affectent ${ }^{28}$. Point d'écarts de plume - plus précisément ici de mise entre guillemets - pour inscrire (prescrire ?) ce qui caractériserait la jouissance sexuelle de la femme, " très différente » de celle de l'homme. Les termes « se toucher ", " auto-affection " féminine ne subissent cette transformation, cette mise en "abyme ", que lorsqu'Irigaray engage les questions du "style " et de l' « écriture ". Comme il a été furtivement indiqué précédemment, c'est à partir du corps sans que ceci soit un simple mouvement, ne s'y réduise - de la femme que naît une toile symbolique finement tissée de métaphores se rapportant à une " essence », un " être », une « spécificité » de «la » femme et de «la » différence sexuelle », ancrée dans « la » sexualité féminine, «la » sexuali « té » féminine. Et dans les quelques propositions qui viennent d'être retenues, l'on peut relever la rencontre/fusion que tente d'opérer Luce Irigaray « entre » la mise en place de ce processus métaphorique et la question du "style » de la femme. Car c'est précisément tout ce qui relève de l' " opération féminine " " (. . . l' " abyme " de la distance, le distancement de la distance, la coupe de l'espacement (. . .) ${ }^{29}$ - qui lui permet de construire, d'édifier ce "pont", ce passage vers ce qui aurait été oublié, réprimé, refoulé, vers ce qui devait rester occulté : « le recouvrement d'une possible opération du féminin dans le langage ${ }^{30}$. On a vu précédemment de quoi procédait ce recouvrement. Peut-on dès lors émettre l'hypothèse que, tout comme Simone de Beauvoir, le projet irigarayien ne soustrait pas la question du «style » de celle du contenu, du sens et qu'ainsi elle demeure tributaire de la différence sexuelle, en tant que celle-ci se joue en rapport avec une spécificité de la sexualité, de la corporéité de la femme en général ? Et que, par conséquent, elle entoure de déterminations cette question, ce qui n'est pas

28. ld., p. 24.

29. J. Derrida, Eperons ...., op. cit., p. 38.

30. L. Irigaray, Ce sexe . . ., op. cit., p. 74. 
rien, compte tenu qu'elle voulait précisément, tout d'abord, que son projet d'écriture soit le détrônement de la domination du signifiant-maître, le phallus, l'abolition de tout rapport entre dominants/tes et dominés/ées dans le langage ? L'articulation du "féminin » et du « masculin» dans le langage semble avoir été prise dans les voiles de la logique du même. Malgré le « style » de l'écrit qui semblait vouloir, pouvoir nous amener bien audelà d'elle. Irigaray aurait-elle " mal-entendu ", " méconnu " le texte derridien? Il est permis ici de le soupçonner sérieusement. Autre pan à ouvrir, à explorer . . .

Département de philosophie Université du Québec à Montréal 